\title{
SEMI-ACTIVE CONTROL OF THE ROCKING MOTION OF \\ MONOLITHIC ART OBJECTS
}

\section{Rosario Ceravolo $^{1^{*}}$, Marica Leonarda Pecorelli ${ }^{1}$, Luca Zanotti Fragonara ${ }^{2}$}

${ }^{1}$ DISEG, Politecnico di Torino, Corso Duca degli Abruzzi 24, 10129, Torino, Italy rosario.ceravolo@polito.it, $\underline{\text { marica.pecorelli@polito.it }}$

2 SATM, Cranfield University, College Road, Cranfield, MK43 OAL, United Kingdom 1.zanottifragonara@cranfield.ac.uk

*Corresponding Author: tel. +390110904821

\section{Abstract}

The seismic behaviour of many art objects and obelisks can be analysed in the context of the seismic response of rigid blocks. Starting from the pioneering works by Housner, a large number of analytical studies of the rigid block dynamics were proposed. In fact, despite its apparent simplicity, the motion of a rigid block involves a number of complex dynamic phenomena such as impacts, sliding, uplift and geometric nonlinearities. While most of the current strategies to avoid toppling consist in preventing rocking motion, in this paper a novel semi-active on-off control strategy for protecting monolithic art objects was investigated. The control procedure under study follows a feedback-feedforward scheme that is realised by switching the stiffness of the anchorages located at the two lower corner of the block between two values. Overturning spectra have been calculated in order to clarify the benefits of applying a semi-active control instead of a passive control strategy. In 
accordance with similar studies, the numerical investigation took into account the dynamic response of blocks with different slenderness and size subject to one-sine pulse excitation.

Keywords: Art objects, rigid block, rocking, semi-active control, non-linear system.

\section{Introduction}

While many studies and codes are devoted to the seismic protection of existing buildings and in particular of museum buildings, the protection of the contents of museums, e.g. art objects on display or kept in storage, has received much less attention by the earthquake engineering standards. This notwithstanding, in the last years the protection of museum collections against seismic hazard is becoming a critical issue.

The past and recent seismic events have clearly shown the high vulnerability of art objects, and of museum contents, even in case of moderate earthquakes. Indeed, the art objects in many museums are displayed so that stability is not ensured during seismic events, and storage areas are often overloaded without any consideration for the seismic risk. The seismic mitigation of art objects requires a multidisciplinary approach in order to find a compromise between safety, conservation and exhibition.

From a structural point of view, the seismic behaviour of art objects can typically be analysed within the context of the dynamic response of rigid bodies. The literature counts a large number of analytical studies on the non-linear dynamics of rigid blocks, starting from the pioneering work of Housner in 1963 [1]. The motion of rigid blocks on a rigid plane can be classified into six types: rest, slide, rotation, slide-rotation, translation jump and rotation jump. The equations of motion, transitions of motion and the motion after the impact between the block and the floor, in the presence of horizontal and vertical accelerations, were investigated by Ishiyama in 1982 [2]. Depending on the form and magnitude of the excitation [2] [3] and on the geometry and mass distribution of the 
objects, the artefacts can experience all the types of motion mentioned above. Among different cases, a particular and fundamental role is assumed by the rocking motion that causes objects to fall from their supports and/or to collide with other objects.

To mitigate the damage due to the rocking motion and to limit the probability of overturning, four different strategies are currently used [4]: (i) lowering the centre of gravity of the artefacts; (ii) adjusting the base-to-height ratio proportions of the art objects; (iii) fixing the objects to the floor/wall and (iv) separating the objects from the ground using base isolation devices. The efficacy of the first two strategies must be attributed to the dynamics of rigid block. During the rocking motion of a rigid block the restoring force is essentially due to its own rotational inertia. Lowering the centre of gravity of an artefact allows to increase, for a given value of the horizontal external action, the ratio between the restoring and the overturning moment of the system, and consequently its stability. The second strategy, i.e. adjusting the base-to-height ratio proportions, can be easily explained in terms of slenderness on overturning, a concept introduced for the first time by Housner [1] and subsequently investigated by several authors [5] [6]. These two strategies, as well as anchoring the objects to the ground, must be used with caution because of the possible high forces transmitted to the artefact. In this case, the art object is forced to bend and deform, instead of oscillating rigidly around the two corners. Hence, failure due to excessive stress is likely, especially in presence of weak and cracked material.

In contrast to the significant amount of theoretical research on the response of free-standing blocks, there are relatively few studies on the response of anchored objects. The in-plane behaviour of a rigid block on a rigid plane anchored with elastic-brittle restraints was studied by Dimentberg et al. [7] and by Makris et al. [8]. In particular Dimentberg et al. investigated the behaviour of anchored blocks excited by white noise, while Makris et al. studied the response of them to pulse-type ground motions, showing that there is a finite frequency range where the conclusions drawn by Dimentberg et al. do not hold concerning the response. The study about pulse-type excitations inferred that a 
frequency range exists where a free-standing block has a better performance than an anchored block.

Passive control strategies were also proposed to reduce the vulnerability of slender rigid blocks. For instance Dimitrakopoulos et al. [9] have proposed anchorages endowed with passive dampers placed at the lower corners of the block. Instead, this paper presents a few investigations concerning semi-active control strategies for blocks anchored with adjustable stiffness. Despite the 3D nature of the real rocking motion, in term of geometry and excitation, the numerical simulation performed, at this stage of the research are limited to a $2 \mathrm{D}$ rocking motion. In more detail, the simulations, conducted for blocks with different size and slenderness, were aimed at evaluating the performance of the restraint system proposed and of the associated control laws. Overturning was also examined in order to draw a few general conclusions.

\section{Model of the block}

Fig. 1(a) depicts a homogeneous rigid block with a rectangular shape in elevation and a rectangular footprint resting on a horizontal plane. The geometry of the block, assuming a 2D motion, is described by a size parameter $\mathrm{R}=\sqrt{b^{2}+h^{2}}$ and by the slenderness $\lambda=b / h$, while the angle $\alpha$, defined as $\alpha=\tan ^{-1}(b / h)$, is the so-called critical angle of the block, i.e. the angle beyond which the overturning of the object will occur under gravity alone.

The rocking motion of a rigid block, disturbed from its equilibrium position, starts with the rotation of the block about one of its corners. If the restoring force is larger than the toppling one, the object will undergo a deceleration in the angular motion, tending to return to the upright position with a residual angular velocity. Due to this residual velocity, the block overshoots the equilibrium position and begins to rotate about the other corner. The change of rotation centre implies an impact between the block and the support and, consequently, energy dissipation and decrease of the block angular velocity. This oscillatory motion continues until the impact will dissipate enough energy to make the magnitude of the overturning moment $M_{O}$ smaller than the restoring one, $M_{R}$. Obviously the rocking motion will stop also if overturning occurs. 


\section{Figure 1 about here}

The governing equations of rocking motion of rigid blocks received a special attention in the literature. Housner [1] defined the governing equations of rocking motion of a free-standing rigid block (Fig. 1(b)) subject to horizontal and vertical base excitations, taking into account non-linear effects. Makris et al. [8] and Dimitrakopoulos et al. [9] modified these equations in order to consider the presence of unilateral elastic-brittle anchorages (Fig. 1(c)) or damper devices (Fig. 1(d)) located at the two bottom corners of the rigid block. A brief summary of these dynamic models is reported hereinafter.

\subsection{Initial response}

Three different types of motion may characterise the initial response of a rigid block on a rigid plane: pure rocking, pure sliding and slide-rock motion [10] [11]. In this work the coefficient of friction between the block and its support is assumed to be sufficiently large so that the object enters in a pure rocking motion without experiencing any sliding. From an analytical point of view, it is also assumed that the following conditions are satisfied by the block (anchored and unanchored) at the initial time instant $t_{0}$ :

$$
\begin{gathered}
\left|m \ddot{X}_{g}\left(t_{0}\right)\right| h>b m\left(g+\ddot{Y}_{g}\left(t_{0}\right)\right) \\
\left|m \ddot{X}_{g}\left(t_{0}\right)\right| \leq \mu_{s} m\left(g+\ddot{Y}_{g}\left(t_{0}\right)\right) \\
\frac{3 \lambda g-3 \lambda \ddot{Y}_{g}\left(t_{0}\right)+4 \ddot{X}_{g}\left(t_{0}\right)+\lambda^{2} \ddot{X}_{g}\left(t_{0}\right)}{3 \lambda \ddot{X}_{g}\left(t_{0}\right)+g+4 \lambda^{2} g-\ddot{Y}_{g}\left(t_{0}\right)-4 \lambda^{2} \ddot{Y}_{g}\left(t_{0}\right)} \leq \mu_{s}
\end{gathered}
$$

where: $m$ is the mass of the block; $g$ is the gravitational acceleration; $\ddot{X}_{g}\left(t_{0}\right)$ is the horizontal ground excitation at time $t_{0} ; \ddot{Y}_{g}\left(t_{0}\right)$ is the vertical ground excitation at the same time instant and $\mu_{s}$ is the coefficient of friction between the block and its support.

In more detail, Eq. (1) describes analytically the assumption that the toppling moment caused by the base excitation at time $t_{0}$ is larger than the resisting moment at the same time. Similarly, Eq. (2) bounds the horizontal inertia force $\left|m \ddot{X}_{g}\left(\mathrm{t}_{0}\right)\right|$ to be smaller than the maximum friction force (no 
sliding motion). Finally, Eq. (3) sets the horizontal reaction $f_{x}\left(t_{0}\right)$ at the contact point (either point 0 or $0^{\prime}$ ) to be smaller than the friction force $\mu_{s} f_{y}\left(t_{0}\right)$. This condition must be satisfied to ensure bonding at the pivoting point and to avoid sliding motion while the block is rotating on a corner (no slide-rock motion). Indeed, the slide-rocking motion occurs when the external force is large enough to cause uplift at one corner, but the friction force at the contact point, equal to $\mu_{S} f_{y}\left(t_{0}\right)$, is insufficient to avoid sliding.

\subsection{Condition to sustain rocking motion}

In order to avoid sliding during the entire duration of the rocking motion, the ratio between horizontal reaction $f_{x}(t)$ and vertical reaction $f_{y}(t)$ exchanged at the contact points (defined as 0 and $0^{\prime}$ in Fig. 1) between the block and the support, always satisfies the following condition:

$$
\left|\frac{f_{x}(t)}{f_{y}(t)}\right|<\mu_{s}
$$

The horizontal and vertical reaction values at the contact points, 0 or $0^{\prime}$, fluctuate with time and they can be derived from the dynamic equilibrium in horizontal and vertical directions:

$$
\begin{gathered}
f_{x}(t)=m\left[\ddot{X}_{g}(t)+\ddot{x}(t)\right] \\
f_{y}(t)=m\left[\ddot{Y}_{g}(t)+g+\ddot{y}(t)\right]
\end{gathered}
$$

where $\ddot{x}(t)$ is the horizontal acceleration and $\ddot{y}(t)$ is the vertical acceleration of the centre of mass of the block.

\subsection{Governing equations of the unanchored rigid block}

The governing equation of motion for the free-standing (FS) rigid object with positive angular rotation can be derived by the rotational equilibrium about the corner 0 :

$$
I_{0} \ddot{\theta}(t)+m R \ddot{X}_{g}(t) \cdot \cos (\alpha-\theta(t))+m\left(g+\ddot{Y}_{g}(t)\right) R \sin (\alpha-\theta(t))=0
$$

where: $I_{0}$ is the moment of inertia of the block about $0 ; R$ is the distance between the centre of rotation and the centre of mass of the block. Similarly, the rocking about $0^{\prime}$ (negative angular rotation) is described by: 


$$
I_{0} \ddot{\theta}(t)+m R \ddot{X}_{g}(t) \cdot \cos (\alpha+\theta)-m\left(g+\ddot{Y}_{g}(t)\right) R \sin (\alpha+\theta(t))=0
$$

By introducing the frequency parameter of the rectangular block, defined as:

$$
p=\sqrt{\frac{m g R}{I_{0}}}=\sqrt{\frac{3 g}{4 R}}
$$

the Eqs. (7) and (8) can be rewritten in a compact form:

$$
\ddot{\theta}(t)+p^{2}\left\{\left(1+\frac{\ddot{Y}_{g}(t)}{g}\right) \sin (\operatorname{sgn}[\theta(t)] \alpha-\theta(t))+\frac{\ddot{X}_{g}(t)}{g} \cos (\operatorname{sgn}[\theta(t)] \alpha-\theta(t))\right\}=0
$$

where sgn[ ] denotes the signum function.

An additional equation, connecting the angular velocities immediately before and after the impacts, must couple with Eq. (10), in order to reproduce the full motion of a rigid block subject to a generic ground excitation.

\subsection{Energy dissipation at impact}

The rotation centre switches from point 0 to 0 ' and vice-versa which implies an impact between the block and its support. During the impact there is energy loss and, consequently, the angular velocity decreases. Housner [1] derived a model to calculate the angular velocity of the rigid body immediately after the impact. This model uses the following assumptions: (i) the impact is punctual; (ii) the impact time $t_{\mathrm{I}}$ is very short; (iii) the block remains in the same position during the impact time. Under these assumptions the relationship between the two angular velocities $\dot{\theta}\left(t_{\mathrm{I}}^{-}\right)$and $\dot{\theta}\left(t_{\mathrm{I}}^{+}\right)$, immediately before the and after the impact, respectively, can be derived from the principle of conservation of the angular moment [12].

The value of the angular momentum immediately before the impact, $M_{A}\left(t_{\mathrm{I}}^{-}\right)$, can be written as function of the horizontal velocity $\dot{u}$ and of the position $u$ at time $t_{\mathrm{I}}^{-}$of the centre of gravity $G$. Having defined the unitary vectors $e_{y}, e_{x}$ and $E_{x}$ as in Fig. 2, the horizontal velocity and the position of $G$ immediately before the impact are expressed as: 


$$
\begin{gathered}
\dot{u}\left(t_{\mathrm{I}}^{-}\right)=R \cdot \dot{\theta}\left(t_{I}^{-}\right) \cdot e_{y} \\
u\left(t_{\mathrm{I}}^{-}\right)=2 \mathrm{~b} \cdot E_{x}+R \cdot e_{\mathrm{x}}
\end{gathered}
$$

whilst, the angular momentum of the block can be written as:

$$
\begin{aligned}
& M_{A}\left(t_{\mathrm{I}}^{-}, O^{\prime}\right)=\int_{V} u\left(t_{\mathrm{I}}^{-}\right) \times \dot{u}\left(t_{\mathrm{I}}^{-}\right) d m= \\
& \quad=\left(\int_{V}\left(R^{2}-2 b R \sin \alpha\right) d m\right) \dot{\theta}\left(t_{I}^{-}\right) \cdot e_{Z}
\end{aligned}
$$

which, after integration, becomes:

$$
M_{A}\left(t_{\mathrm{I}}^{-}, O^{\prime}\right)=m R^{2}\left(\frac{4}{3}-2 \sin ^{2} \alpha\right) \dot{\theta}\left(t_{I}^{-}\right) \cdot e_{z}
$$

Similarly, having defined the position and the horizontal velocity of the block immediately after the impact as:

$$
\begin{gathered}
\dot{u}\left(t_{I}^{+}\right)=R \cdot \dot{\theta}\left(t_{I}^{+}\right) \cdot e_{y} \\
u\left(t_{I}^{+}\right)=R \cdot e_{x}
\end{gathered}
$$

the angular momentum of the block becomes:

$$
M_{A}\left(t_{I}^{+}, O^{\prime}\right)=\left(\int_{V} R^{2} d m\right) \dot{\theta}\left(t_{I}^{+}\right) \cdot e_{z}=\frac{4}{3} m R^{2} \dot{\theta}\left(t_{I}^{+}\right) \cdot e_{z}
$$

Equalling Eqs. (14) and (17), a relationship between the velocity immediately before and after the impact is found:

$$
\frac{\dot{\theta}\left(t_{I}^{+}\right)}{\dot{\theta}\left(t_{I}^{-}\right)}=1-\frac{3}{2} \sin ^{2} \alpha=e
$$

In the literature $e$ is referred to as coefficient of restitution and it is related to the energy $E_{L}$ lost at time impact given by the following equation:

$$
E_{L}=\frac{1}{2} I_{0} \dot{\theta}\left(t_{I}^{+}\right)-\frac{1}{2} I_{0} \dot{\theta}\left(t_{I}^{-}\right)=\frac{2}{3} m R^{2}\left[\dot{\theta}^{2}\left(t_{I}^{+}\right)-\dot{\theta}^{2}\left(t_{I}^{-}\right)\right]=\frac{2}{3} m R^{2}\left(1-e^{2}\right) \dot{\theta}^{2}\left(t_{I}^{-}\right)
$$

In this study, it is assumed that the coefficient of restitution is exactly described by Eq. (19). However, in real practice, a discrepancy is observed between the experimental values of $e$ and the 
theoretical one of Eq. (19). Some studies have stressed a dependence of $e$ on the interface materials [13], the amplitude of oscillation [14] and the velocity immediately before the impacts [15]. Therefore, the real values of the coefficient of restitution can be smaller or larger than the theoretical one [13] [15] [16] [17].

In order to evaluate whether after the impact the object will rest or will undergo uplift on the other corner, the absolute values of overturning moment $M_{\mathrm{O}}$ and restoring moment $M_{R}$ should be compared. The object will rest if the following condition is satisfied:

$$
\left|M_{R}\right|>\left|M_{\mathrm{O}}\right|
$$

Excluding bouncing, at time impact the inertia of the body contribute to overturning moment, the self-weight to restoring one while the external excitation can intensify the overturning or the restoring moment depending on its direction.

\subsection{Governing equations of the anchored rigid block}

The governing equations of the block with unilateral elastic-brittle constant anchorages with stiffness $K(\mathrm{EBK})$, see Fig. 1(c), differ from those of the free-standing block due to the presence of an additional restoring moment. Fig. 3(c) illustrates the moment-rotation relation for the EBK block, as resulting from the moment-rotation relation of the FS block (Fig. 3(a)) plus the contribution of the elastic-brittle anchorages (Fig. 3(b)).

Figure 3 about here

The restoring moment due to the elastic anchorages with respect to the rotation centre is:

$$
M_{K}(\theta)=K \cdot 4 b \sin \left|\frac{\theta(t)}{2}\right| \cdot 2 b \cos \left|\frac{\theta(t)}{2}\right|=K \cdot 4 R^{2} \sin ^{2} \alpha \sin |\theta(t)|
$$

and it turns out to be equal to zero when the rotation of the block is null $(\sin |\theta(t)|=0)$.

Consequently, the compact form of the governing equation for the anchored block becomes: 


$$
\begin{aligned}
\ddot{\theta}(t)+p^{2}\{(1+ & \left.\frac{\ddot{Y}_{g}(t)}{g}\right) \sin (\operatorname{sgn}[\theta(t)] \alpha-\theta(t))+\frac{\ddot{X}_{g}(t)}{g} \cos (\operatorname{sgn}[\theta(t)] \alpha-\theta(t)) \\
& \left.+\frac{M_{K}(\theta)}{I_{0} p^{2}} f_{K, L 1}\left(\theta_{\max }(\mathrm{t})\right) f_{K, L 2}(\theta(t))+\frac{M_{K}(\theta)}{I_{0} p^{2}} f_{K, R 1}\left(\theta_{\min }(\mathrm{t})\right) f_{K, R 2}(\theta(t))\right\}
\end{aligned}
$$

where: $f_{K, R 1}\left(\theta_{\min }(\mathrm{t})\right)$ and $f_{K, L 1}\left(\theta_{\max }(\mathrm{t})\right)$ are fracture functions for the right and left elasticbrittle anchorages, respectively, whereas $f_{K, R 2}(\theta(t))$ and $f_{K, L 2}(\theta(t))$ are two functions used to establish which of the two anchorages is working during the rocking motion.

$\theta_{\min }$ defines the maximum negative rotation that the block reached at the generic time $t, \theta_{\max }$ is the maximum positive rotation at the same time and $\left|\theta_{f}\right|$ is the rotation angle corresponding to the collapse of the working anchorage, and the fracture functions can be defined as:

$$
\begin{gathered}
f_{K, R 1}\left(\theta_{\min }(\mathrm{t})\right)=\left\{\begin{array}{lll}
1 & \text { if } & \theta_{\min }(\mathrm{t})<-\theta_{f} \\
0 & \text { if } & \theta_{\min }(\mathrm{t}) \geq-\theta_{f}
\end{array}\right. \\
f_{K, L 1}\left(\theta_{\max }(\mathrm{t})\right)=\left\{\begin{array}{lll}
1 & \text { if } & \theta_{\max }(\mathrm{t})<\theta_{f} \\
0 & \text { if } & \theta_{\max }(\mathrm{t}) \geq \theta_{f}
\end{array}\right.
\end{gathered}
$$

Similarly, $f_{K, R 2}(\theta)$ and $f_{K, L 2}(\theta)$ read:

$$
\begin{aligned}
& f_{K, R 2}(\theta(t))=\left(\frac{1-\operatorname{sgn}[\theta(t)]}{2}\right) \\
& f_{K, L 2}(\theta(t))=\left(\frac{1+\operatorname{sgn}[\theta(t)]}{2}\right)
\end{aligned}
$$

Eq. (21) reflects a large displacement formulation, accounting for a spring elongation equal to $4 b \sin |\theta(t) / 2|$ and an eccentricity of $2 b \cos |\theta(t) / 2|$ with respect to the rotation corner.

The formulation of the problem for restraints with adjustable stiffness remains unchanged. The only difference with respect to the block with constant stiffness is that $K$ in Eq. (21) is not constant with time and may change in accordance with the control law.

\subsection{Governing equations of the anchored rigid block with dampers}

The governing equation of motion of a rigid block anchored with viscous damper devices (VDD) placed at two corners of the block can be obtained, similarly to the previous case, from Eq. (10) by 
adding the restoring moment due to the working viscous damper device (see Fig. 1(d)). The restoring moment of the damper devices around the corner of rotation reads:

$$
M_{D}(\theta)=D\left(2 b \cos \left|\frac{\theta(t)}{2}\right| \dot{\theta}(t)\right)\left(2 b \cos \left|\frac{\theta(t)}{2}\right|\right)=D 4 b^{2} \cos ^{2}\left|\frac{\theta(t)}{2}\right| \dot{\theta}(t)
$$

where $D$ is the damping parameter of the devices. Eq. (10) can be rewritten as:

$$
\begin{aligned}
\ddot{\theta}(t)+p^{2}\{(1+ & \left.\left.\frac{\ddot{Y}_{g}(t)}{g}\right) \sin (\operatorname{sgn}[\theta(t)] \alpha-\theta(t))+\frac{\ddot{X}_{g}(t)}{g} \cos (\operatorname{sgn}[\theta(t)] \alpha-\theta(t))\right\} \\
& +\frac{M_{D}(\theta)}{I_{0}} f_{D, L 1}\left(\theta_{\max }(\mathrm{t})\right) f_{D, L 2}(\dot{\theta}(t))+\frac{M_{D}(\theta)}{I_{0}} f_{D, R 1}\left(\theta_{\min }(\mathrm{t})\right) f_{D, R 2}(\dot{\theta}(t)) \\
& =0
\end{aligned}
$$

where $f_{D, R 1}\left(\theta_{\min }(\mathrm{t})\right)$ and $f_{D, L 1}\left(\theta_{\max }(\mathrm{t})\right)$ are fracture functions for the right and left damper devices, respectively, whereas $f_{D, R 2}(\theta(t))$ and $f_{D, L 2}(\theta(t))$ are functions used to establish which of the two devices is working during the rocking motion. Similarly to the formulation of the EBK block, $f_{D, R 1}(\theta(t))$ and $f_{D, L 1}(\theta(t))$ are defined as:

$$
\begin{gathered}
f_{D, R 1}\left(\theta_{\min }(t)\right)=\left\{\begin{array}{lll}
1 & \text { if } & \theta_{\min }(t)<-\theta_{f} \\
0 & \text { if } & \theta_{\min }(t) \geq-\theta_{f}
\end{array}\right. \\
f_{D, L 1}\left(\theta_{\max }(t)\right)=\left\{\begin{array}{lll}
1 & \text { if } & \theta_{\max }(t)<\theta_{f} \\
0 & \text { if } & \theta_{\max }(t) \geq \theta_{f}
\end{array}\right.
\end{gathered}
$$

whereas $f_{D, R 2}(\theta(t))$ and $f_{D, L 2}(\theta(t))$ are defined as:

$$
\begin{aligned}
& f_{D, R 2}(\theta(t))=\left(\frac{1-\operatorname{sgn}[\theta(t)]}{2}\right) \\
& f_{D, L 2}(\theta(t))=\left(\frac{1+\operatorname{sgn}[\theta(t)]}{2}\right)
\end{aligned}
$$

The models described hereinabove are inherently nonlinear. In fact, there are four different causes of nonlinearity in the rocking motion of a rigid block: the change of rotation centre from one edge to the other (implying a change of equation of motion); the impact energy dissipation (which induces a jump discontinuity in the angular velocity); the geometric effect of the slenderness ratio of the object (which implies the presence of geometric nonlinearity terms in the governing equation) and the coupling of the vertical excitation with the rocking response (entailing a change of conditions to 
$\overline{\text { initialize and sustain pure rocking motion). These different sources of nonlinearity were investigated }}$ by several authors [18] [19]. In addition to this, the presence of the "on-off" stiffness introduces a further source of non-linearity in the rocking motion problem.

\section{Algorithm and control laws}

\subsection{Algorithm description}

Closed form solutions for the rocking response to simple excitation forms are only found in the small displacement range. Therefore, the large displacement response of the rigid block subject to one-sine pulse has been solved numerically in Matlab®, by integrating Eqs. (10), (22) and (28) through a fourth-order Runge-Kutta scheme. The time-step used in the numerical integration was set to $0.0025 \mathrm{~s}$.

Starting from the rest state of the rigid block, the actual corner of rotation, and the equation to integrate, is determined using the sign of the horizontal base excitation. In fact, for a positive value of $\ddot{X}_{g}\left(t_{0}\right)$, the block starts to rotate around the left corner (negative rotation), while for a negative value of horizontal ground acceleration it rotates around the right corner (positive rotation). The same equation is used for subsequent time steps until the sign of the rotation $\theta$ changes. At each step, the condition $\theta(t-1) \cdot \theta(t) \geq 0$ is checked in order to establish a possible change of rotation corner. If the condition is not satisfied, the velocity immediately before the impact, $\dot{\theta}\left(t_{\mathrm{I}}^{-}\right)$, is evaluated and the velocity just after the impact, $\dot{\theta}\left(t_{\mathrm{I}}^{+}\right)$, is determined by means the Eq. (18). With the initial conditions $\theta\left(t_{I}\right)=0$ and $\dot{\theta}\left(t_{I}\right)=\dot{\theta}\left(t_{I}^{+}\right)$the equation of motion on the other corner is used to evaluate the motion of the block in the subsequent time interval until the rotation angle $\theta$ will change again its sign. The numerical code has been validated by comparing its solutions with time-histories reported for the same blocks and excitations by Housner [1], Makris et al. [20] and Spanos et al. [21]. 


\subsection{Control laws}

The stiffness of the control system is assumed to switch between two fix values, $K_{\max }$ and $K_{\min }$, as a function of the state of the rigid block and of the ground excitation. With the aim to limit the rotation of the block, two different control laws have been examined. In both cases the stiffness at the generic time $t+1$ is assigned as a function of the current state of the block and, for the second law, also as a function of the sign of the ground excitation.

The first control law (CL01) minimises the positive work done by the anchorages and maximises the negative one. Therefore, the stiffness value of the restraint system during the rocking motion is instantaneously assigned in accordance with the following law:

$$
K(t+1)=\left\{\begin{array}{lll}
K_{\max } & \text { if } & \theta(t) \cdot \dot{\theta}(t) \geq 0 \\
K_{\min } & \text { if } & \theta(t) \cdot \dot{\theta}(t)<0
\end{array}\right.
$$

The second control law (CL02) considered has two different working modes for setting the value of $K(t+1)$. The first modality consists in minimising the absolute value of the total work, and it applies when the block is excited. The second one, which applies in the free oscillations conditions, consists in minimising the work done by the anchorage, this time in its signed value. Therefore, during the excitation, the stiffness is assigned using the following law:

$$
K(t+1)=\left\{\begin{array}{lll}
K_{\max } & \text { if } & \ddot{X}_{g}(t) \cdot \theta(t) \leq 0 \\
K_{\min } & \text { if } & \ddot{X}_{g}(t) \cdot \theta(t)>0
\end{array}\right.
$$

In the free oscillation phase, this control law coincides with the one in Eq. (33).

Fig. 4 and Fig. 5 depict the normalised time-history of values assumed by the two "on-off" anchorages, located at the two lower corners of the block (see Fig. 1(c)), in accordance with the two control laws examined. The block characteristics and the excitations are the same. It is worth noticing that the stiffness of the anchorage at the rotation pole is ineffective, thus a zero value was assigned in the plots.

Figure 4 about here

Figure 5 about here 
Due to the difference between the positive and the negative work done by the anchorages, this type of restraint system dissipates additional energy to that dissipated by impacts.

In Fig. 6 the phase portrait of the block anchored with the "on-off" elastic brittle anchorages is compared with that of the same block equipped with viscous damper devices. To stress the capability of the restraint system to dissipate energy, the coefficient of restitution $r$ was assumed equal to one. Therefore, the spiral-shaped representation of cycles is to be attributed only to the dissipative characteristics of the restraint systems of the block.

Figure 6 about here

The dissipative properties of the restraint system depend not only on the control law but also on the values attributed to $K_{\max }$ and $K_{\min }$. In fact, since the dissipated energy increases with increasing $K_{\max }$ and reducing $K_{\min }$, these two values must be chosen as a function of the technology available and of the resistance of the artefact in correspondence of the anchorages. Fig. 7 shows the responses of a rigid block using the CL02 control law and for different values of $K_{\min }$ and $K_{\max }$.

Figure 7 about here

In a real practice implementation, the spring model must be intended as a system able to exert a time-varying restraint force that depends on the measured value of the uplift. Consequently, an equivalent implementation of the proposed control concept consists in replacing the physical springs with magnetic devices that can be regulated via a current.

\section{Numerical validation of the semi-active control strategy}

This section reports a few examples of the control strategy applied to rigid blocks subject to onesine pulse excitations, characterised by different amplitudes, $a_{p 0}$, and frequencies, $\omega_{p}$. Studying rigid blocks under one-sine excitation constitutes the first step towards the control under a generic loading (e.g. earthquake). 
To start with, overturning spectra were utilised to clarify the benefits of semi-active control with respect to viscous damper devices or to anchorages with constant stiffness $K$. The numerical investigations regarded the dynamic response of 9 blocks with different size $(R)$ and slenderness $(\lambda)$ (see Table 1).

Table 1 about here

To evaluate the performance of the "on-off" semi-active control, two systems were compared: (i) a block with constant stiffness $K$, such that $K=K_{\max }$; (ii) a viscously damped block, with the damping parameter $D$ chosen so that its free decay response, in the assumption of no dissipation at impacts $(r=1)$, fits the phase portrait of the same block subjected to semi-active control (see Fig. $6)$.

The first assumption implies the same behaviour for the two blocks (EBK block and OOK block) during their initial stages of motion (see Fig. 8(a)), thus allowing to appreciate the effect of the "onoff" control.

The second condition implies that the amount of energy dissipated by the "on-off" control is comparable with the one dissipated by the same block with damper devices (see Fig. 8(b)). However, it is worth underlining that the performance of the control is not to be measured in terms of mere dissipation, as it reflects a different strategy to prevent overturning.

Figure 8 about here

In accordance with previous studies [22], $K_{\max }$, and thus $K$, are chosen so that the failure strength of the anchorages, $F_{f}$, which is set at $0.4 \mathrm{mg}$, corresponds exactly to the critical angle $\alpha$ of the blocks. As for $K_{\min }$, its value is set to zero (on-off control).

Regarding the 9 VDD blocks, the damping, obtained from the curve fitting equivalence, resulted in similar values for systems having the same frequency parameter $p$. The values of relative damping, defined as $\gamma=3 D \sin ^{2} \alpha / 2 m p$, are reported in Table 2 for the investigated blocks. 
Table 2 about here

\subsection{Simulation results}

The overturning spectra associated to the 9 blocks are obtained by calculating the responses to one-sine pulses characterised by a frequency, $w_{n}$, in the range $(p, 6 p)$ and an amplitude, $a_{p o}$, in the range $(1.1 g \cdot \alpha, 10 g \cdot \alpha)$. The ranges were chosen so as to appreciate the effect of the anchor devices and of the control strategies on the overturning boundaries. It is worth noticing that in such plots the domain of interest depends on the block slenderness and size. As an example, for a pulse with amplitude and frequency of $1 \mathrm{~g}$ and $10 \mathrm{~Hz}$ respectively, the maximum significant ratio $a_{p 0} / \alpha g$ can be assumed approximately equal to the block slenderness. Similarly, for blocks with $R$ equal to $1.8394,0.8175$ and $0.4598 \mathrm{~m}$ (see Table 1 ), the significant values for $w_{n} / p$ can be virtually limited to 31,20 and 15 , respectively.

For each couple of values, $w_{n}$ and $a_{p o}$, a point of the safe or unsafe area of overturning spectra is obtained, depending to whether or not overturning occurs.

From numerical simulations a substantial overlapping of the overturning spectra for blocks with the same slenderness and different size was found, independently of the restraint conditions (FS, EBK, etc.). For example, Fig. 9(a) superimposes the overturning spectra of two free-standing blocks, (BLC02 and BLC05) having the same slenderness but different size (see Table 1 for more details). The unsafe area of the BLC05, defined by the magenta boundary, is seen to fit approximately the unsafe area of the BLC02 identified by the dotted area. One may notice that Fig. 9(a) conveys information only on the occurrence of toppling, while nothing can be inferred about the actual nature of the responses. Indeed, the rotation time-histories of the two blocks subject to the same excitation $\left(w_{n} / p=5\right.$ and $\left.a_{p 0} / \alpha g=2\right)$ present a difference in form of a stretching of the response in the time axis (see comparison in Fig. 9(b)) .

Makris et al. [8] introduced a useful representation in terms of overturning spectra of blocks. Similarly, the overturning spectra reported in Fig. 9(a) identify two different unsafe regions, in 
accordance with previous works [8] [20][22]: (i) unsafe area, mode 1, corresponding to overturning after one impact and (ii) unsafe area, mode 2, corresponding to overturning without impact.

Figure 9 about here

Based on the previous comments to Fig. 9(a), the results referred to three blocks with the same size $(R)$ and different slenderness $(\lambda)$, BLC04, BLC05 and BLC06, are reported in order to evaluate the performance of the semi-active control strategies.

Fig. 10 depicts the overturning spectra of three blocks controlled according the two laws in Eq. (33) and Eq. (34) (CL01 and CL02, respectively).

Figure 10 about here

Fig. 10 shows that for all the blocks the use of semi-active control, independently of the control law utilised, has no effect on the domain associated to overturning without impact (mode 2). Instead, real time adjustment of the anchorages stiffness affects the extension of the unsafe area referred to the first overturning mode. From Fig. 10 one may observe the superiority in performance of the second control law (CL2). Indeed, the clear reduction in the unsafe area supports a feedbackfeedforward control strategy, which entails the measurement of both input and output.

The performance of the "on-off" semi-active control was then compared with that of uncontrolled blocks, namely free-standing blocks, anchored blocks with additional constant stiffness and anchored blocks with viscous damper devices. The overturning spectra, obtained for the different restraint conditions, are superimposed in Fig. 11, 12 and 13. From the same figures one can observe that the second overturning mode is not significantly influenced by the type of connection to the ground, as in this case the overturn occurs for amplitude values that depend only on the excitation frequency. On the contrary, the first overturning mode appears to be considerably influenced by the characteristics of the anchorages.

Figure 11 about here 


\section{Figure 12 about here}

Figure 13 about here

The use of a semi-active control sensibly reduces the extension of the unsafe area associated to overturn with impact (Fig. 11, 12 and 13) without the negative effects pointed up by Makris et al. [8] due to the introduction of additional stiffness in the block system. Indeed, unlike the case of blocks with constant anchorages stiffness, the unsafe area of the overturning spectra related to the proposed control is enclosed in the unsafe area of the free-standing ones. This occurs independently of the slenderness of the blocks.

Fig. 15 presents a few time-histories of the block BLC04 with different ground connections (FS, KC, VDD and OOK) subject to one-sine pulses (see Fig. 14). In these cases, the anchorage with adjustable stiffness resulted to be the only one that prevented overturning.

Figure 14 about here

Figure 15 about here

Finally, the semi-active control strategy was applied to a real artefact, San Matteo sculpture, allocated in the "Galleria dei Prigioni" in Florence. Starting from the geometric characteristic of Michelangelo's sculpture, the dimension and the mass of the equivalent symmetric block were determined (see Fig. 16(b)). Fig. 16(d) depicts the dynamic responses of equivalent block subject to the same one-sine pulse, evaluated with regard different ground connection. The example reported shows, in accordance with the previous study, that the controlled system is the only one that avoids overturning.

Figure 16 about here

\section{Conclusions}

This paper investigates a strategy for controlling the rocking response of art objects and structures 
that can be approximated as rigid blocks. The assumed control devices consist in adaptive anchorages connected to the ground, whose stiffness can be adjusted according to simple energetic laws. The performance of the semi-active control system was evaluated based on the dynamic behaviour of 9 blocks, with different size and slenderness, subjected to one-sine pulse excitations. Comparisons were performed essentially in term of overturning spectra, as the maximum stress transmitted to the art objects can be limited to acceptable values at the design stage, by choosing the range of the on-off control force.

Overturning spectra were built in order to clarify the benefits of applying a semi-active control, and to draw a few general conclusions on the quality of the proposed control strategy. To this aim, a few comparisons were made with non-controlled systems, including blocks with constant linear springs and damper devices. Then, numerical investigations were conducted on blocks with different size and slenderness. Finally, the control strategy was validated on an equivalent system simulating the S. Matteo statue by Michelangelo.

Numerical simulations led to the following conclusions:

- Control laws that can support a feedback-feedforward control strategy perform better than simple feedback approaches.

- The use of a semi-active control reduces appreciably the extension of the unsafe area associated to overturn with impact (mode 1), without the negative effects due to the introduction of additional stiffness in the block system pointed up by previous works.

- The second mode (overturning without impact) does not seem to be significantly influenced by the type of control, as in this case overturn occurs for amplitude values that depend only on the loading frequency.

Future research will address possible adaptations of the control strategies in order to deal with more general excitation forms, including real ground motions and higher frequency vibrations. 


\section{References}

[1] G. W. Housner, The behaviour of inverted pendulum structures during earthquake. Bulletin of the Seismological Society of America 53 (1963) 403-417.

[2] Y. Ishiyama, Motion of the rigid bodies and criteria for overturning by earthquake excitations. Earthquake Engineering and Structural Dynamics 10 (1982) 635-650.

[3] T. Taniguchi, Non-linear response analyses of rectangular rigid bodies subjected to horizontal and vertical motion. Earthquake Engineering and Structural Dynamics 31 (2002) 1481-1500.

[4] M. Lowry, B.J. Farrar, D. Armendariz, J. Podary, Protecting collection in the J. Paul Getty Museum from earthquake damage, WAAC Newsletter, vol. 29, no. 3, pp. 16-23, September 2007.

[5] C.S. Yim, A.K. Chopra, J. Penzien, Rocking response of rigid blocks to earthquake. Earthquake Engineering and Structural Dynamics 8 (1980) 565-587.

[6] A.N. Kounadis, Parametric study in rocking instability of rigid block under harmonic ground pulse: a unified approach. Soil Dynamics and Earthquake Engineering 45 (2013) 125-143.

[7] M.F. Dimentberg, Y.M. Lin, R. Zhang, Toppling of computer-type equipment under base excitation. Journal of Engineering Mechanics 119 (1993) 145-160.

[8] N. Makris, J. Zhang, Rocking response of anchored blocks under pulse-type motions. Journal of Engineering Mechanics 127 (2001) 484-493.

[9] E.G. Dimitrakopoulos, M.J. DeJong, Overturning of retrofitted rocking structures under pulse-type excitations. Journal of Engineering Mechanics 138 (2012) 963-972.

[10] H.W. Shenton, Criteria for initiation of slide, rock and slide-rosck rigid body modes. Journal of Engineering Mechanics 122 (1996) 690-693.

[11] A. Pompei, A. Scalia, M.A. Sumbatyan, Dynamic of rigid block due to horizontal ground motion. Journal of Engineering Mechanics 124 (1998) 713-717.

[12] A.N. Kounadis, On the overturning instability of a rectangular rigid block under ground excitation. The Open Mechanics Journal 4 (2010) 43-57.

[13] M.A. ElGawady, Q. Ma, J. Butterworth, J.M. Ingham, Probabilistic Analysis of Rocking Blocks, in New Zealand Society for Earthquake Engineering, 2006.

[14] H. Schau, M. Johannes, Rocking and sliding of unanchored bodies subjected to seismic load according to conventional and nuclear rules, in Computational Methods in Structural Dynamics and earthquake Engineering, Kos Island, Greece, 2013.

[15] Q.T. Ming Ma, The mechanics of rocking structures subjected to ground motion, Ph.D. Thesis, 2010.

[16] F. Pena, F. Prieto, P.B. Lourenco, A. Campos Costa, J.V. Lemos, On the dynamics of rocking motion of single rigid-block structures. Earthquake Engineering and Structural Dynamics 36 (2007) 2383-2399.

[17] M. Aslam, W. G. Godden, Earthquake Rocking Response of rigid. Journal of the Structural Division 106 (1980) $377-392$.

[18] S.C.S. Yim, H. Lin, Nonlinear impact and chaotic response of slender rocking objects. Journal of Engineering Mechanics 117 (1991) 2079-2100.

[19] F. Prieto, P.B. Lourenco, On the rocking behaviour of rigid objects. Meccanica 40 (2005) 121-133.

[20] N. Makris, Y. Roussos, Rocking response and overturning of equipment under horizontal pulse-type motions. Pacific Earthquake Engineering Research 5 (1998).

[21] P.D. Spanos, A.S. Koh, Rocking of rigid block due to harmonic shaking. Journal of Engineering Mechanics 110 (1984) 1627 1642.

[22] R. Ceravolo, M.L. Pecorelli, L. Zanotti Fragonara, Adaptive control of the rocking motion of art objects, in Computational Methods in Structural Dynamic and Earthquake Engineering (COMPDYN), Crete Island, Greece, 2015. 
(a)

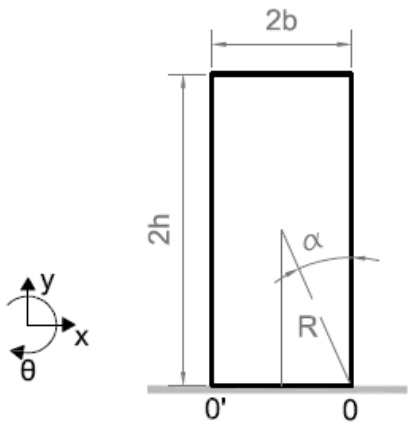

(c)

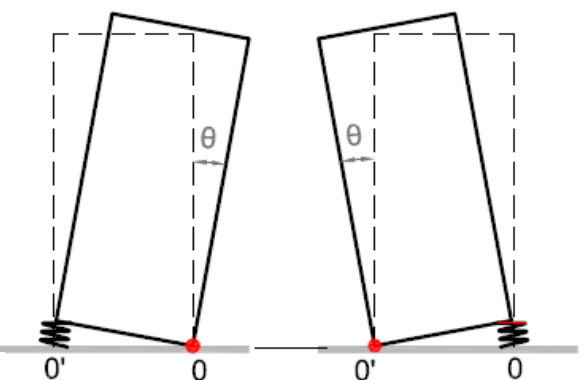

(b)
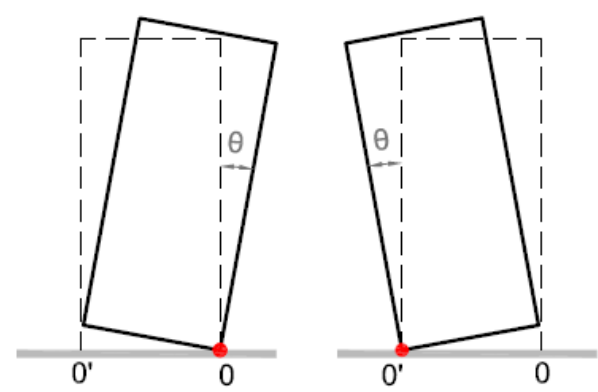

(d)

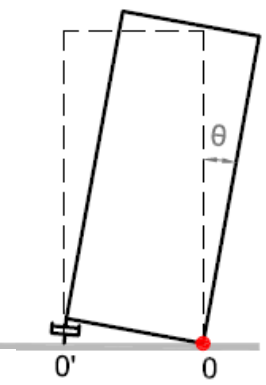

Fig.1. (a) Idealization of an artefact as a rigid body, positive convention of displacements and geometrical parameters of the block; (b) mechanical model of unanchored rigid block; (c) mechanical model of rigid block anchored with unilateral elastic-brittle anchorages (reacting only in tension); (d) mechanical model of rigid block anchored with damper devices. 


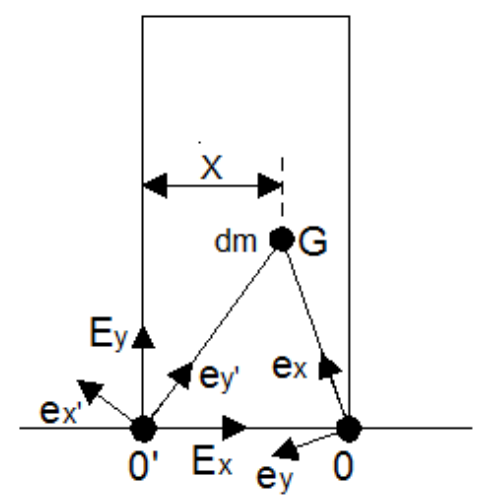

Fig. 2. Rectangular block under rocking motion just before it impacts on point 0 '. 

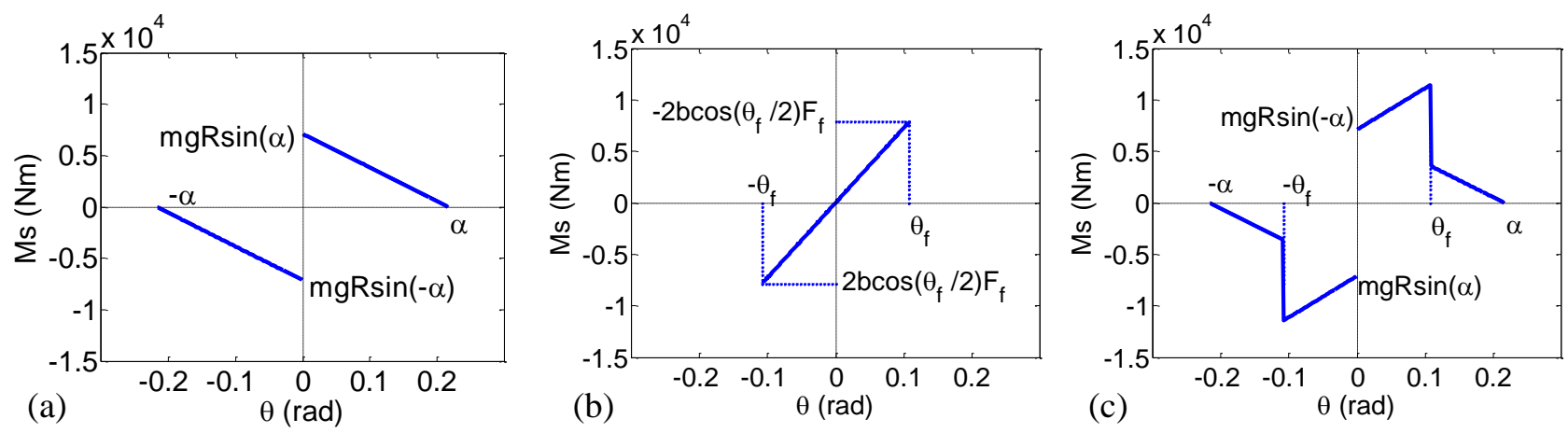

Fig.3. Restoring moment-rotation law. (a) FS block; (b) elastic-brittle anchorages; (c) EBK block. 
(a)

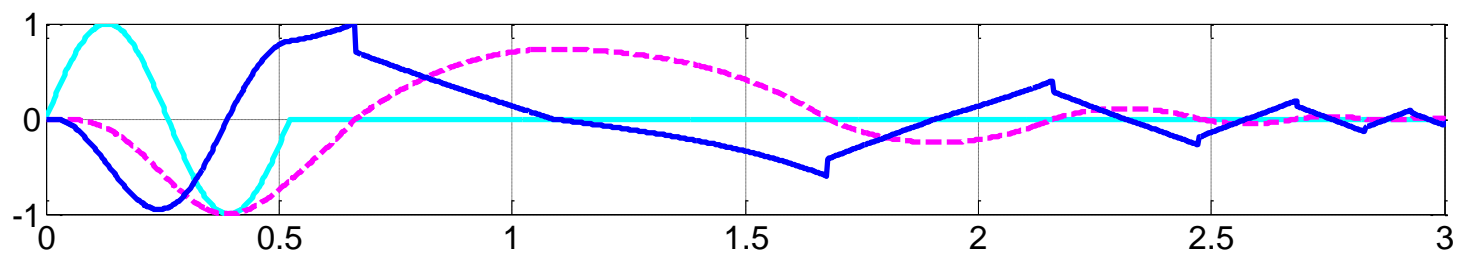

(b)

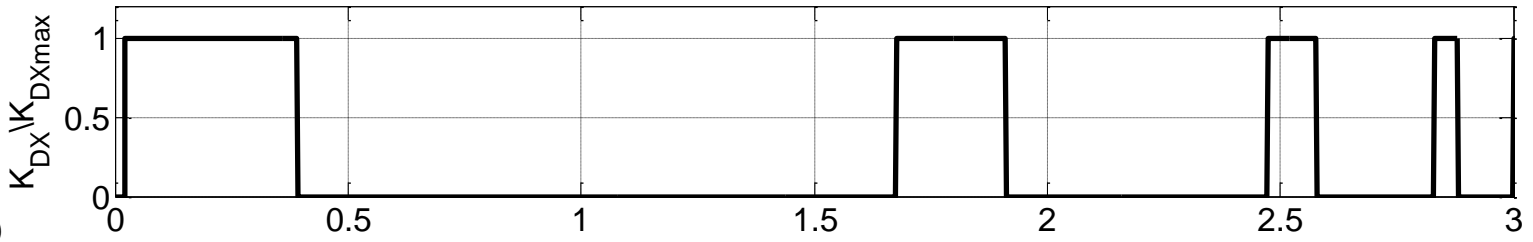

(c)

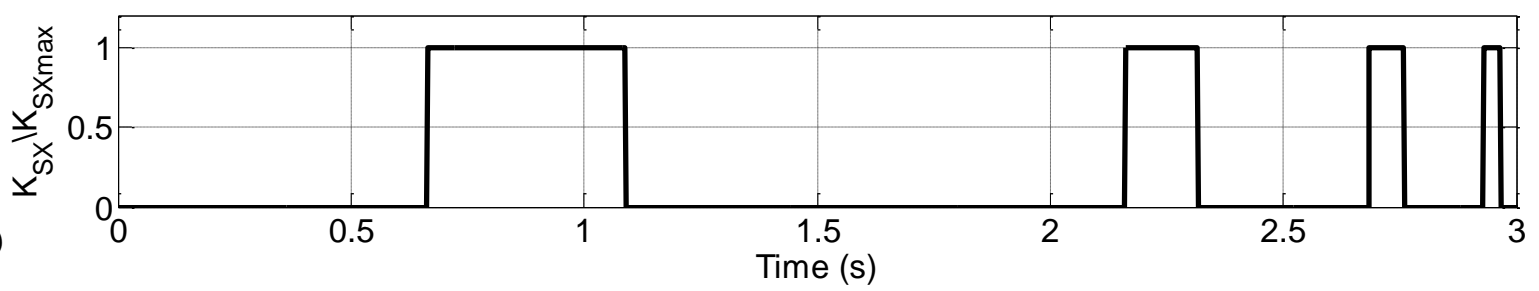

Fig.4. First control law. (a) Normalised time-history of ground motion - , rotation angle $---=$ and angular velocity - of a block with on-off ancorages; (b) normalised time-history of the stiffness of the anchorage placed at the right corner of the block; (c) normalised time-history of the stiffness of the anchorage placed at the left corner of the block. 
(a)
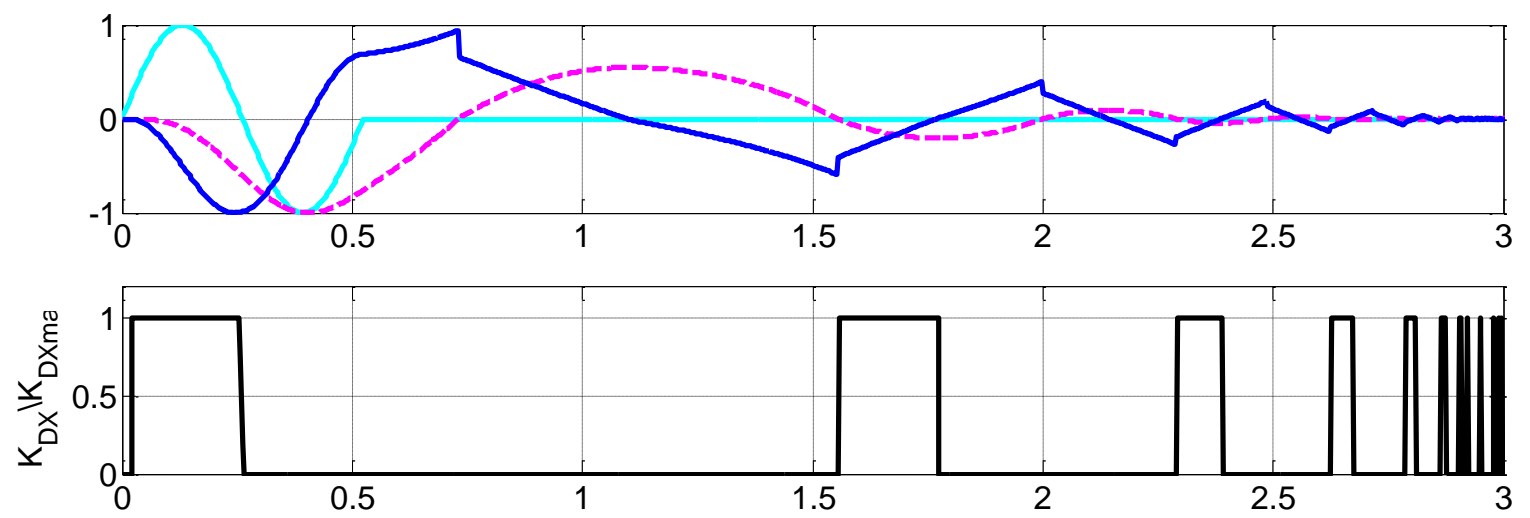

(b)

(c)

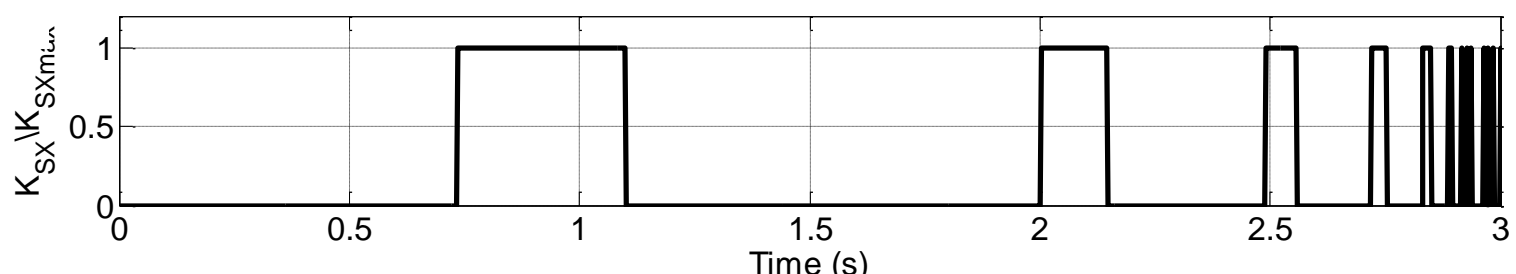

Fig.5. Second control law. (a) Normalised time-history of ground motion — angular velocity — of a block with on-off anchorages; (b) normalised time-history of the stiffness of the anchorage placed at the right corner of the block; (c) normalised time-history of the stiffness of the anchorage placed at the left corner of the block. 

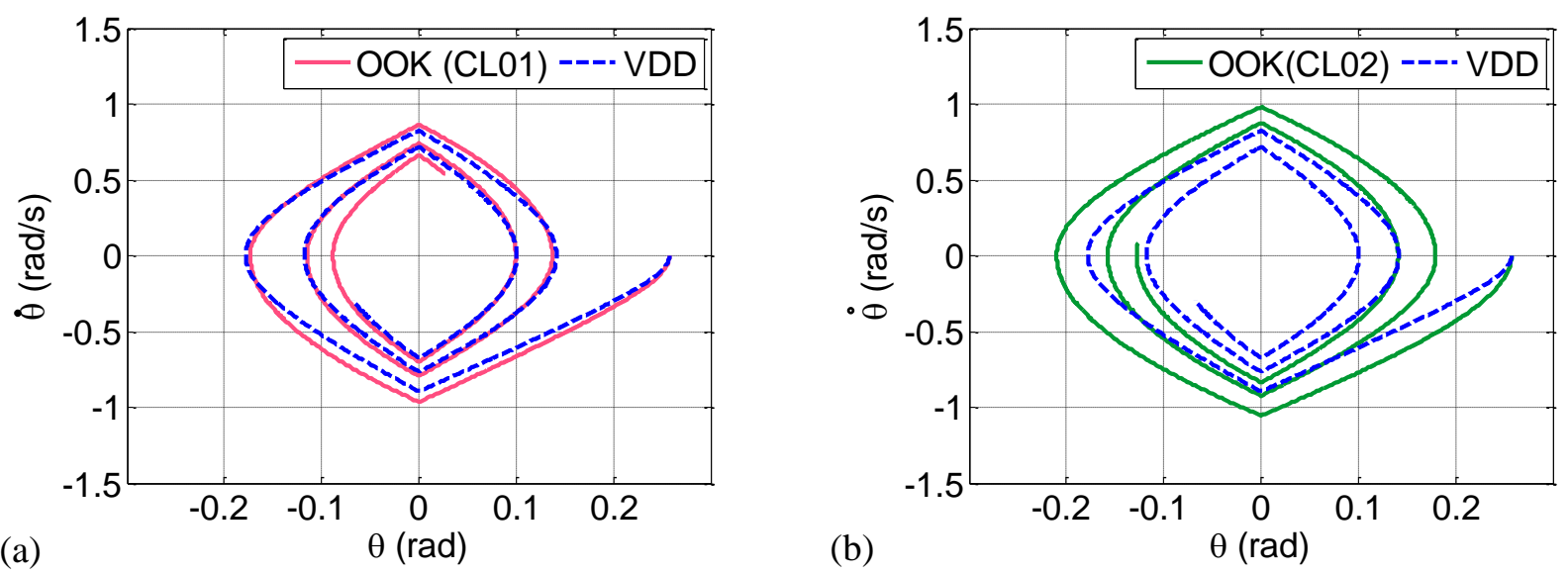

Fig.6. Phase portrait of the free rocking, initial condition $\theta\left(t_{0}\right)=\alpha$ and $\dot{\theta}\left(t_{0}\right)=0$, of a rigid block $(\lambda=3$ and $R=0.8175 \mathrm{~m}$ ) with $r=1$ in case of: (a) controlled block by means of control law CL01 (OOK_CL01) and uncontrolled block equipped with viscous damper devices (VDD); (b) controlled block by means of control law CL02 (OOK_CL02) and uncontrolled block equipped with viscous damper devices (VDD). 

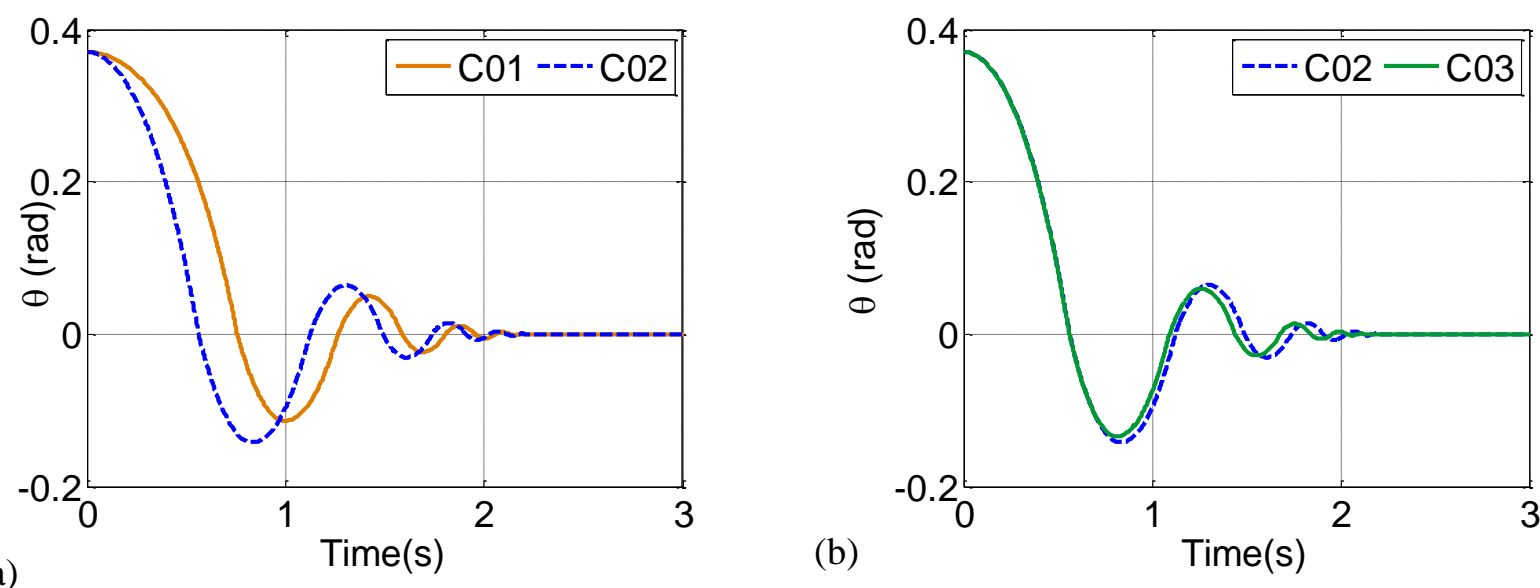

Fig.7. Time-history of OOK block, control law CL02 and different values of $K_{\max }$ and $K_{\min } \mathrm{CS} 01\left(K_{\max }=\right.$ $0.4 \mathrm{mg}, K_{\min }=0 ; \operatorname{CS} 02\left(K_{\max }=0.4 \mathrm{mg}, K_{\min }=0.2 \mathrm{mg}\right) ; \operatorname{CS} 03\left(K_{\max }=0.6 \mathrm{mg}, K_{\min }=0.2 \mathrm{mg}\right)$. 

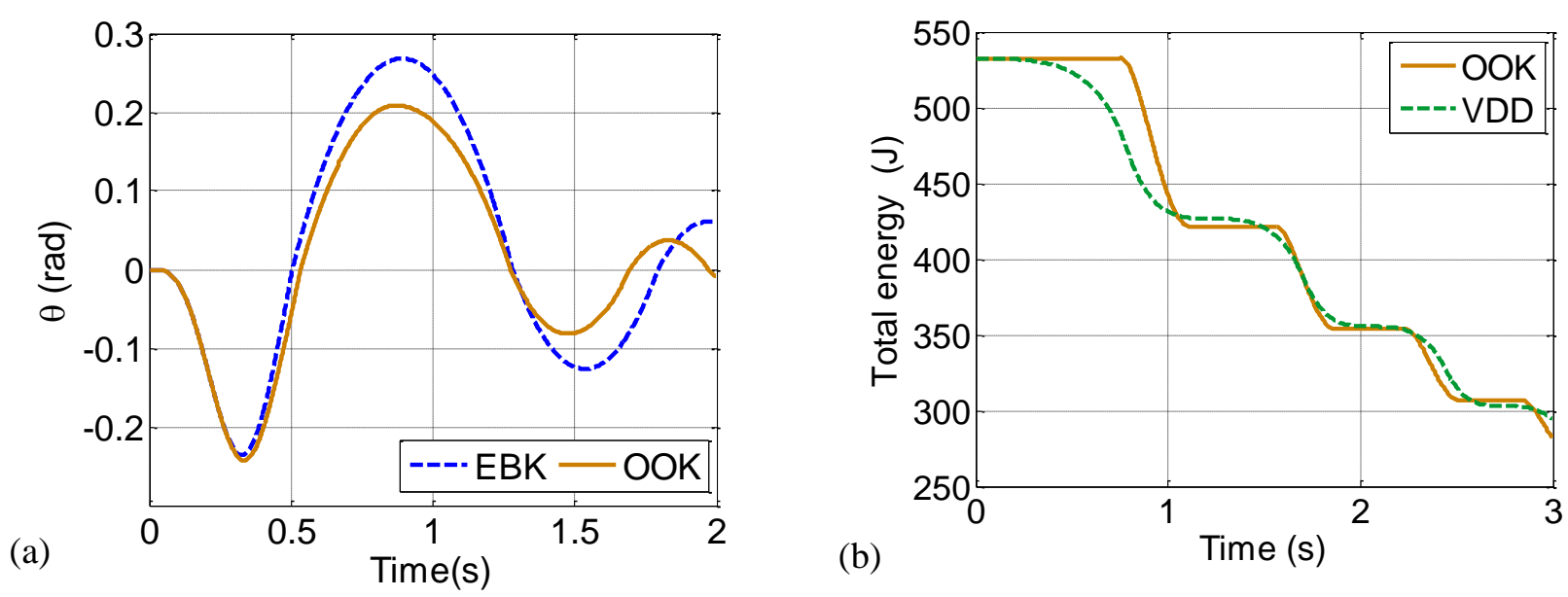

Fig.8. (a) Time-history of a rigid block (BLC05) anchored with constant (EBK) and on-off restraint systems (OOK); (b) time-history of the total energy of a rigid block (BLC05) with on-off restraint system (OOK) and with damper device (VDD). 

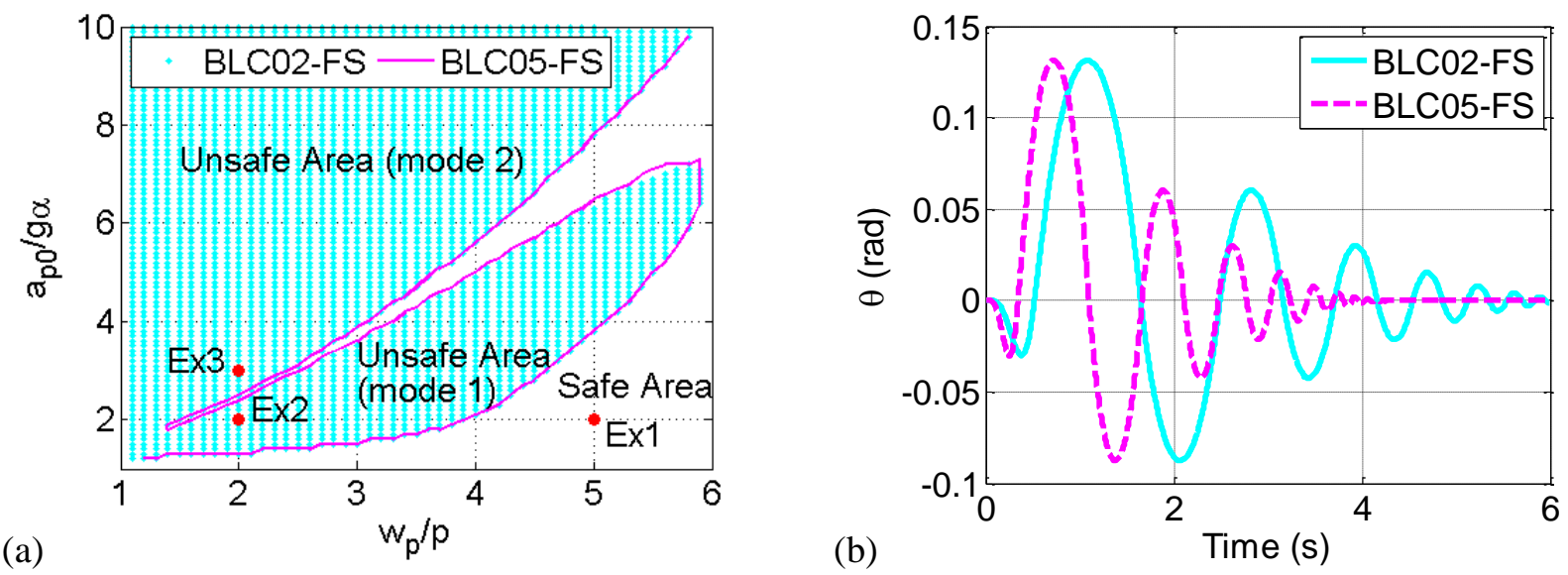

Fig.9. (a) Overlapping of the overturning spectra of blocks (BLC02 and BLC05) having the same slenderness and different size; (b) comparison of time-histories of rigid blocks (BLC02 and BLC05) subject to the same one-sine pulse, "Ex1" in Fig. 9(a) $\left(w_{n} / p=5\right.$ and $\left.a_{p 0} / \alpha g=2\right)$. 
(a)
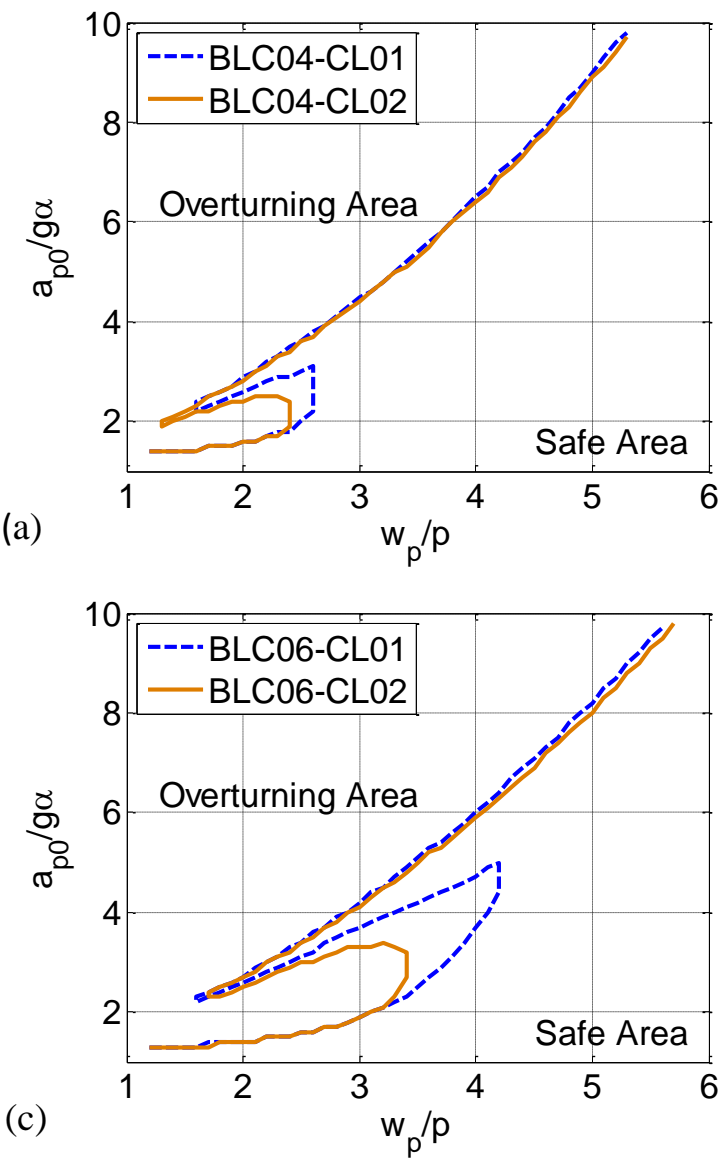

(b)

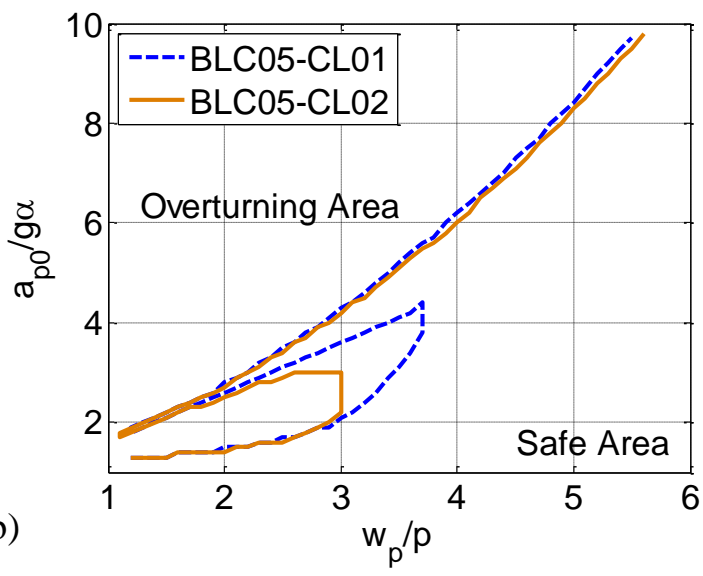

(d)

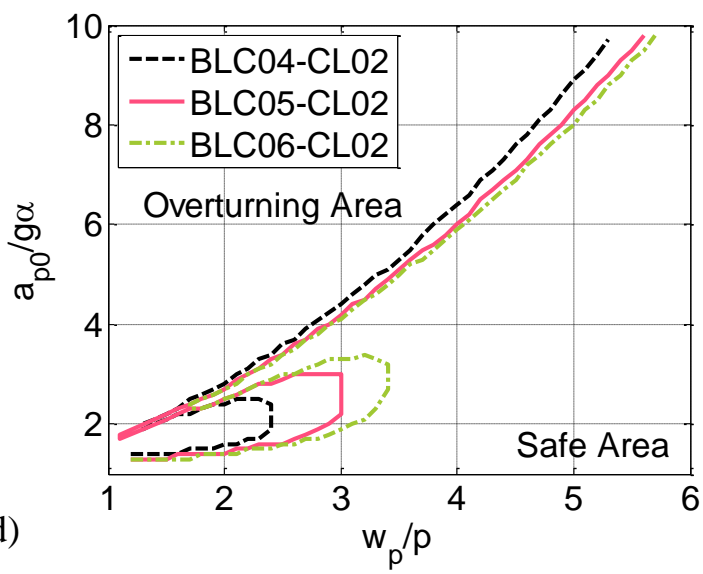

Fig.10. Overturning spectra associated to the two control laws CL01 and CL02: (a) BLC04; (b) BLC05; (c) BLC06 and (d) overturning spectra of the three blocks evaluated by using CL02. 

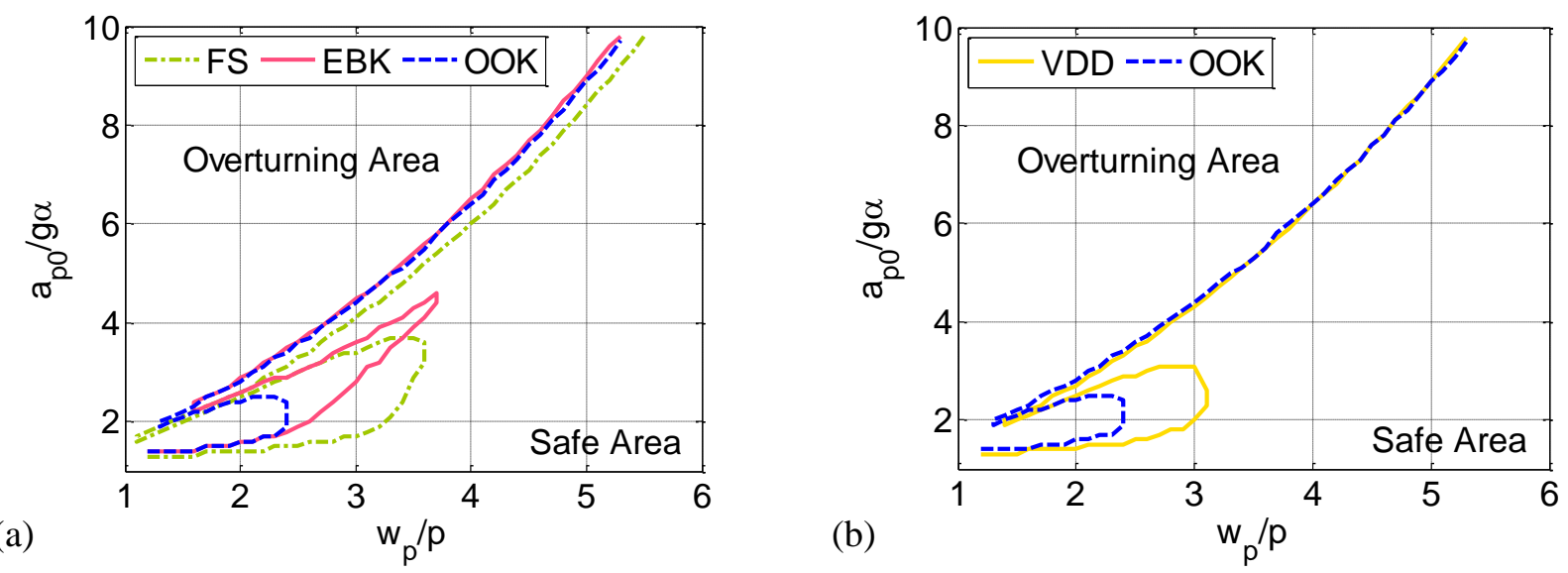

Fig.11. Overturning spectra of BLC04 evaluated for different restraint conditions: free-standing (FS); anchorages with elastic-brittle stiffness (EBK); anchorages with on-off stiffness (OOK); viscous damper devices (VDD). 

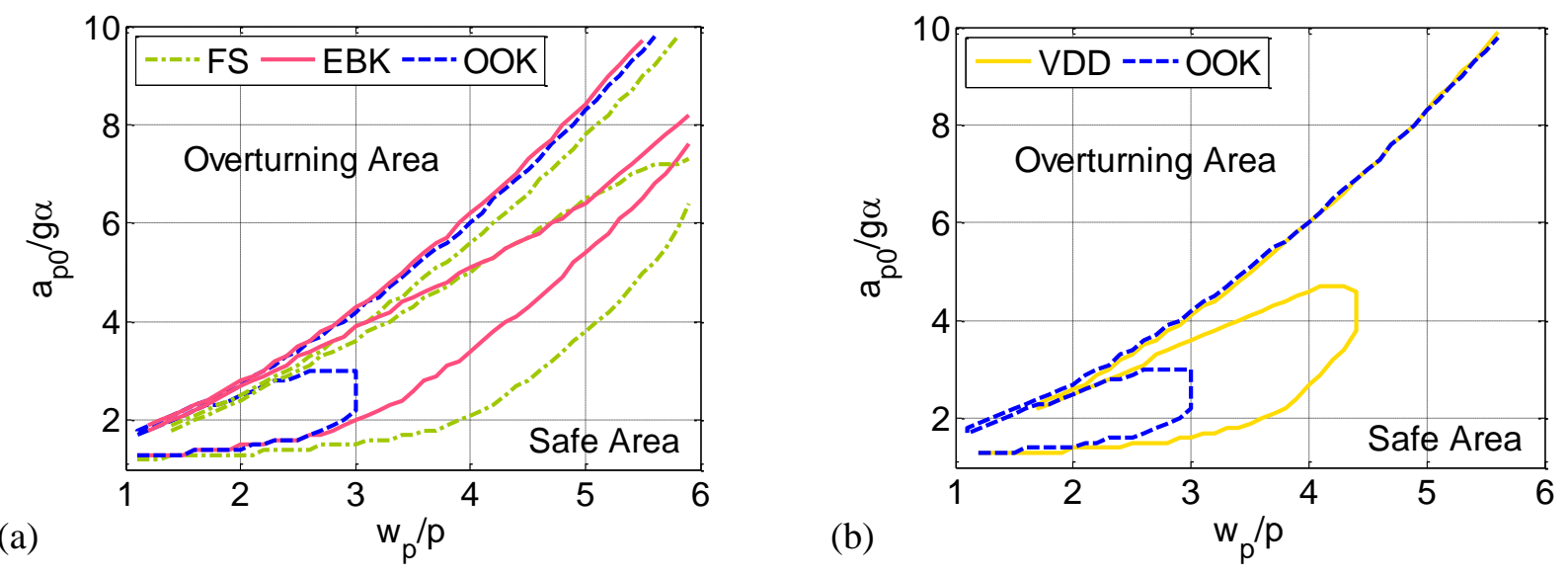

Fig.12. Overturning spectra of BLC05 evaluated for different restraint conditions: free-standing (FS); anchorages with elastic-brittle stiffness (EBK); anchorages with on-off stiffness (OOK); viscous damper devices (VDD). 

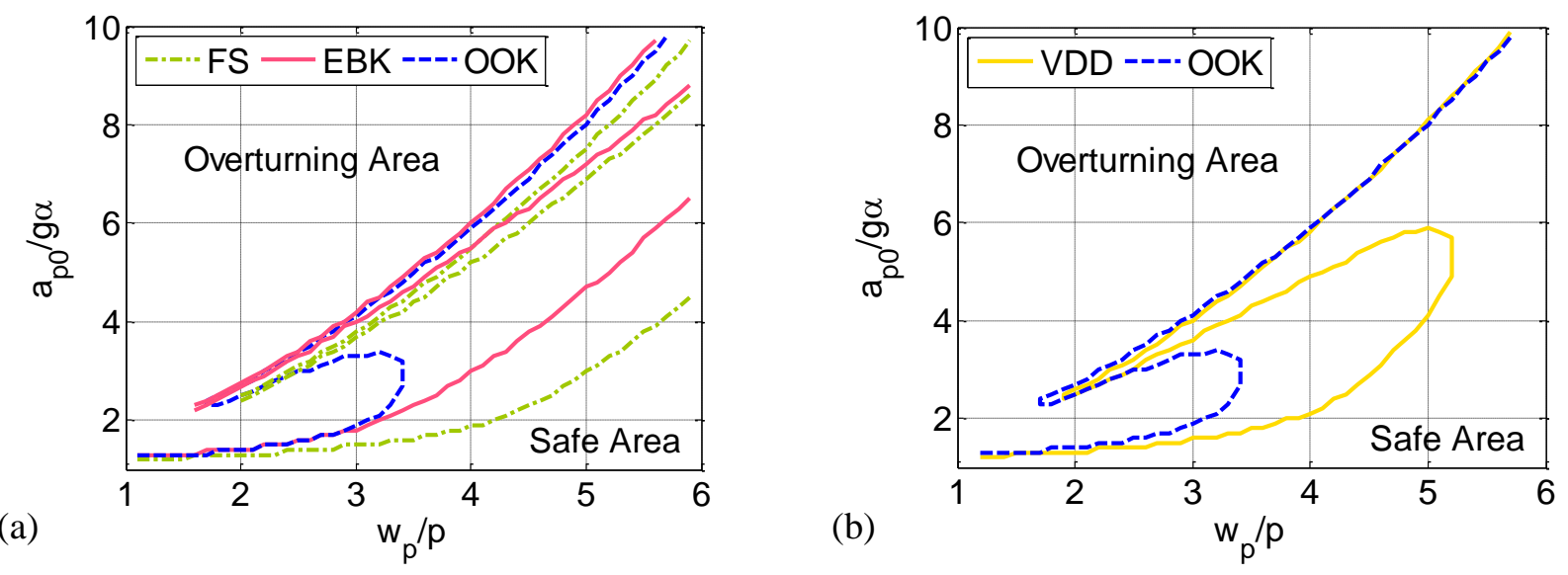

Fig.13. Overturning spectra of BLC06 evaluated for different restraint conditions: free-standing (FS); anchorages with elastic-brittle stiffness (EBK); anchorages with on-off stiffness (OOK); viscous damper devices (VDD). 


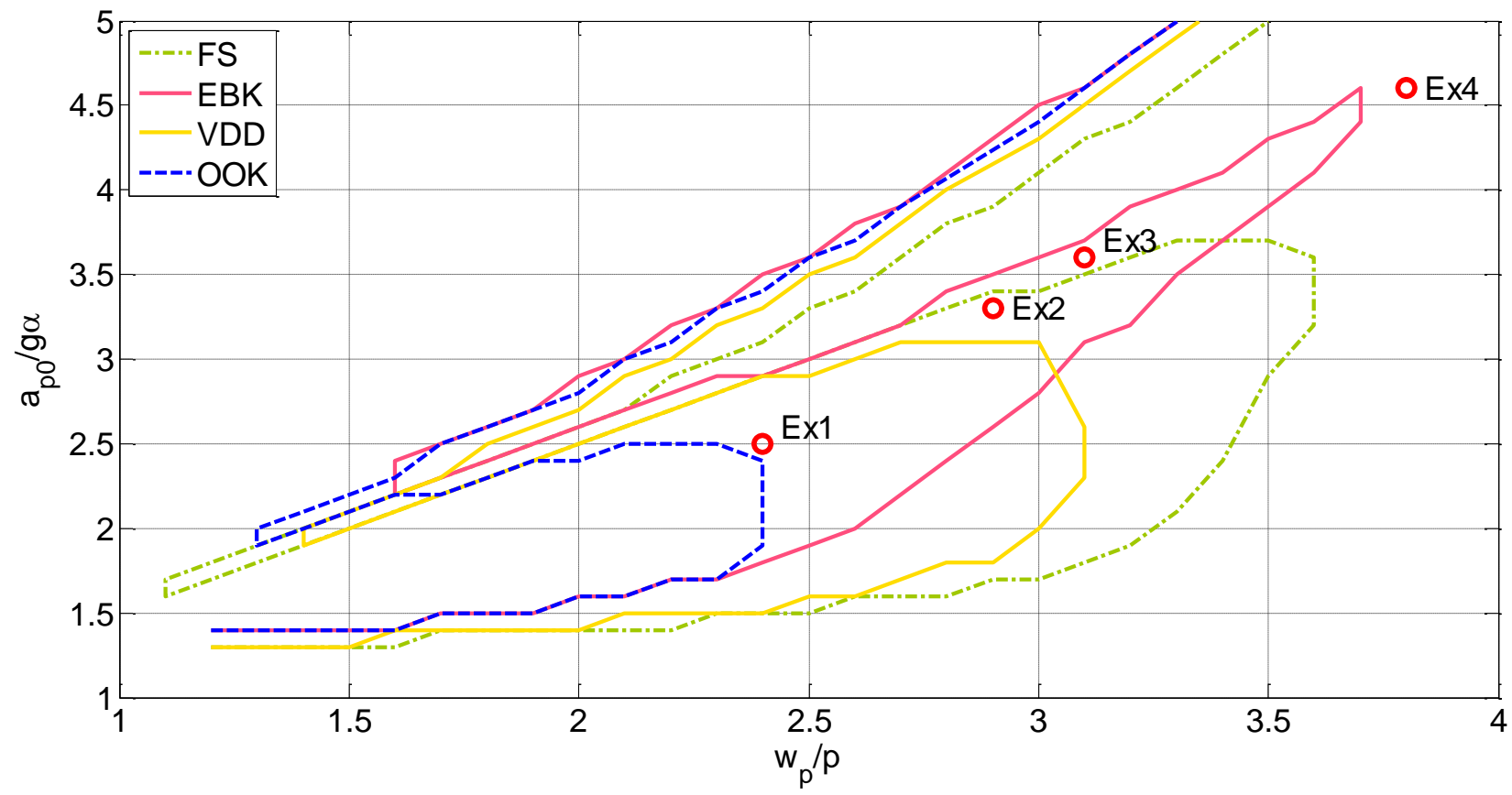

Fig.14. Overturning spectra of BLC04. 


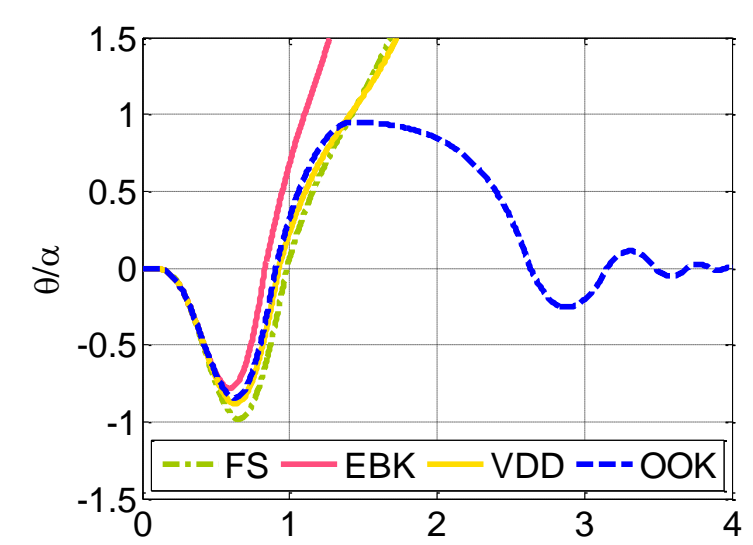

(a)

Time (s)

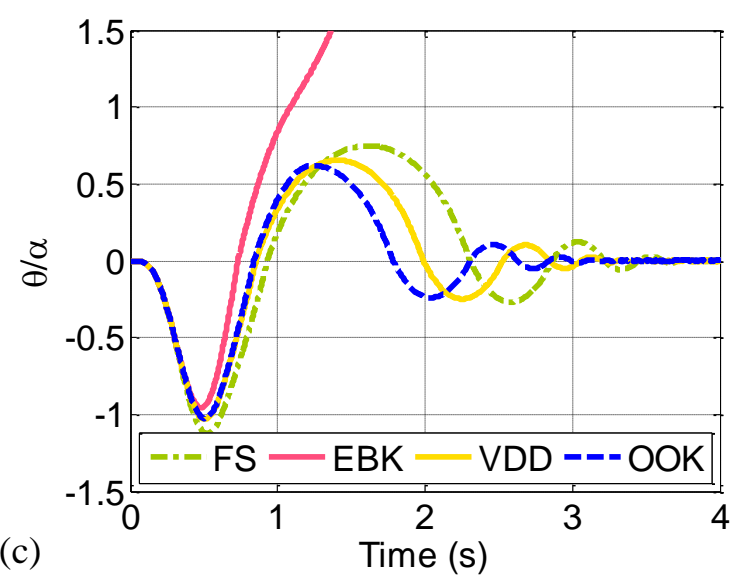

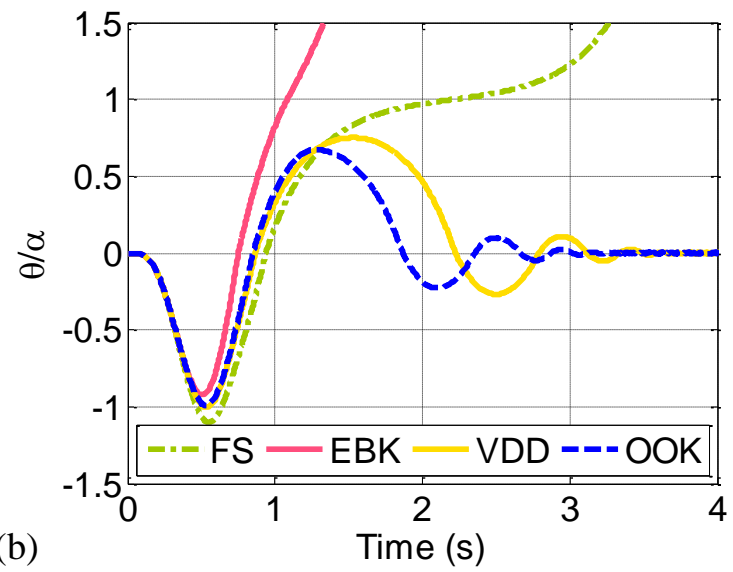

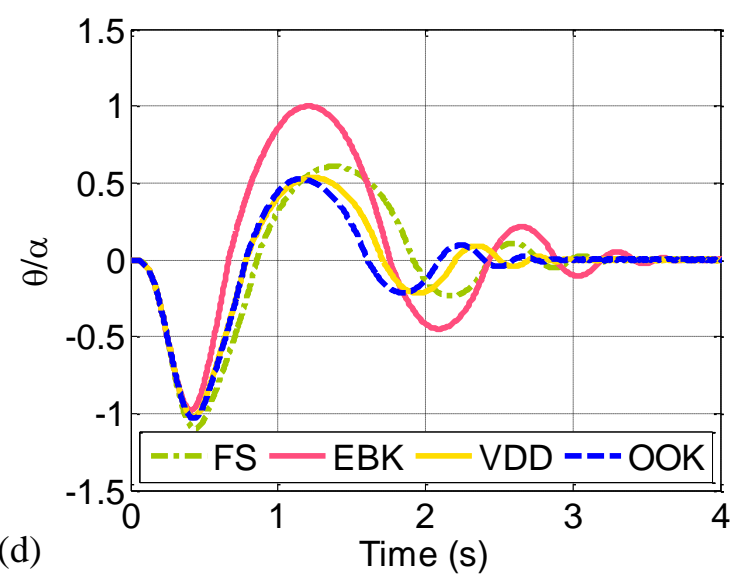

Fig.15. Comparison of normalised time-history of BLC04 with different ground connection subjected to onesine pulse (a) $w_{n} / p=2.4$ and $a_{p o} / g \alpha=2.5$ (excitation "Ex1" in Fig.15); (b) $w_{n} / p=2.9$ and $a_{p o} / g \alpha=3.3$ (excitation "Ex2" in Fig.15); (c) $w_{n} / p=3.1$ and $a_{p o} / g \alpha=3.6$ (excitation "Ex3" in Fig.15); (d) $w_{n} / p=3.8$ and $a_{p o} / g \alpha=4.6$ (excitation "Ex4" in Fig.15). 
2016-04-12

\section{Semi-active control of the rocking motion of monolithic art objects}

Ceravolo, Rosario

Elsevier

Rosario Ceravolo, Marica Leonarda Pecorelli, Luca Zanotti Fragonara, Semi-active control of the rocking motion of monolithic art objects, Journal of Sound and Vibration, Volume 374, 21 July 2016, pp1-16

http://dx.doi.org/10.1016/j.jsv.2016.03.038

Downloaded from Cranfield Library Services E-Repository 